\title{
Properties of non-relativistic string theory
}

\author{
E. A. Bergshoeff, ${ }^{\dagger}$, J. Lahnsteiner ${ }^{\ddagger}$, L. Romano ${ }^{\circ}$ and C. Şimşek ${ }^{\star}$ \\ Van Swinderen Institute, University of Groningen, \\ Nijenborgh 4, 9747 AG Groningen, The Netherlands \\ $\dagger$ e.a.bergshoeff@rug.nl, $\$$ jo.lahnsteiner@hotmail.com, \\ - lucaromano2607@gmail.com, ᄎ c.simsek@rug.nl
}

4th International Conference on Holography, String Theory and Discrete Approach

Hanoi, Vietnam, 2020 doi:10.21468/SciPostPhysProc.4

\begin{abstract}
We show how Newton-Cartan geometry can be generalized to String Newton-Cartan geometry which is the geometry underlying non-relativistic string theory. Several salient properties of non-relativistic string theory in this geometric background are presented and a discussion of possible research for the future is outlined.
\end{abstract}

(c) (i) Copyright E. A. Bergshoeff et al. This work is licensed under the Creative Commons Attribution 4.0 International License. Published by the SciPost Foundation.
Received 28-10-2020

Accepted 22-12-2020

Published 13-08-2021

doi:10.21468/SciPostPhysProc.4.001

\section{Introduction}

Starting from classical mechanics, there are at least three interesting ways to extend the theory each of which introduces a constant of nature that is absent in classical mechanics: (1) at large velocities with respect to the velocity of light $c$ the theory extends to special relativity; (2) at small distances certain physical quantities get quantized in units of the reduced Planck's constant $\hbar$ corresponding to quantum mechanics and (3) a gravitational force can be introduced via Newton's constant $G$ leading to Newtonian gravity. There are two well-known ways to combine two of these extensions: (1) extending classical mechanics with high velocities and gravity leads to general relativity and (2) extending classical mechanics to high velocities and small distances leads to quantum field theory. Logically speaking, however, there is a third way, namely extending classical mechanics to small distances and gravity. This would lead to a theory of non-relativistic (NR) quantum gravity. Finally, the maximal extension to high velocities, small distances and gravity leads to the long sought for theory of quantum gravity. This situation can nicely be summarized via the the so-called Bronstein cube [1] in Figure 1.

Usually, the issue of finding a consistent theory of quantum gravity is approached either by adding gravity to quantum field theory or by quantizing general relativity. The Bronstein cube suggests a third way to approach this issue: can quantum gravity be viewed as the relativistic extension of a self-consistent NR theory of quantum gravity? This leads to the related question 


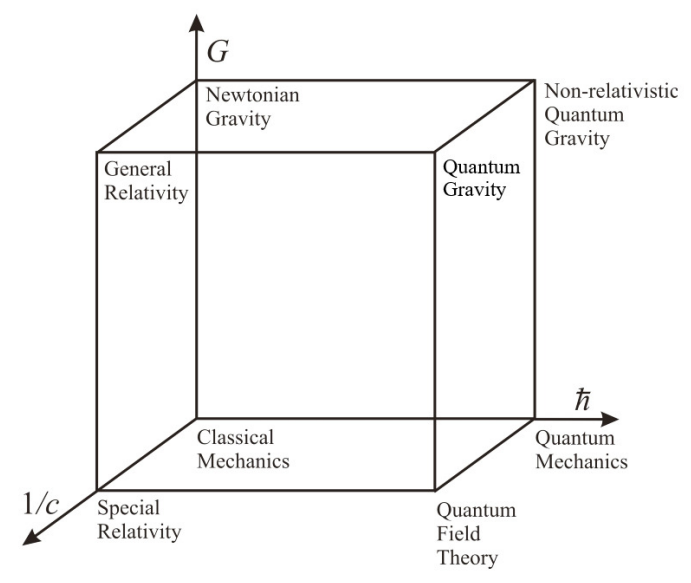

Figure 1: The Bronstein cube shows how classical mechanics can be extended in three different ways to (1) special relativity, (2) quantum mechanics and (3) Newtonian gravity. Combining two of these extensions leads to general relativity, quantum field theory or NR quantum gravity. Ultimately, combining all three extensions leads to relativistic quantum gravity.

of how essential relativity is in constructing a theory of quantum gravity or, put differently, whether one can take in a consistent way the NR limit of quantum gravity. Motivated by this we wish to address the following intriguing question:

can one define a consistent NR theory of quantum gravity?

This question can be asked for each approach to define a consistent theory of quantum gravity: is relativity essential for the construction, yes or no? String theory is one approach to define a theory of quantum gravity. In this talk we wish to discuss the definition of a NR string theory including its underlying geometry and some of its basic properties. In particular, we will show how the geometry corresponding to NR string theory can be viewed as a generalization of the well-known Newton-Cartan (NC) geometry that underlies NC gravity.

\section{From NC Gravity to String NC Gravity}

The independent fields of $D$-dimensional NC geometry are given by $(a=1, \cdots, D-1)$

$$
\left\{\tau_{\mu}, E_{\mu}{ }^{a}, M_{\mu}\right\}
$$

Here, $\tau_{\mu}$ is the time-like Vierbein acting as the clock function and $E_{\mu}{ }^{a}$ is the spatial Vierbein acting as the ruler. The charge corresponding to the gauge field $M_{\mu}$ is a central charge in the Galilei algebra thereby extending it to the Bargmann algebra. These gauge fields transform under (local) spatial rotations with parameters $\lambda^{a}{ }_{b}$, Galilean boosts with parameters $\lambda^{a}$ and central charge transformations with parameter $\sigma$ as follows:

$$
\begin{aligned}
\delta \tau_{\mu} & =0, \\
\delta E_{\mu}{ }^{a} & =\lambda^{a}{ }_{b} E_{\mu}{ }^{b}+\lambda^{a} \tau_{\mu}, \\
\delta M_{\mu} & =\partial_{\mu} \sigma+\lambda_{a} E_{\mu}{ }^{a} .
\end{aligned}
$$


The spin-connection fields $\omega_{\mu}^{a b}$ corresponding to spatial rotations and $\omega_{\mu}{ }^{a}$ corresponding to Galilean boosts are functions of $\tau_{\mu}, E_{\mu}{ }^{a}$ and $M_{\mu}$.

In NC gravity one cannot define a single non-degenerate metric for the full spacetime like the Riemannian metric in general relativity. Instead, one defines two degenerate metrics

$$
\tau_{\mu \nu}=\tau_{\mu} \tau_{v} \quad \text { and } \quad h^{\mu \nu}=E_{a}^{\mu} E_{b}^{v} \delta^{a b}
$$

that are invariant under the Bargmann transformations (2). Here $E_{a}^{\mu}$ is the projective inverse of $E_{\mu}{ }^{a}$ which, unlike the spatial Vierbein, is invariant under Galilean boosts. This means that the combination

$$
E_{\mu}{ }^{a} E_{\nu}^{b} \delta_{a b}
$$

is not invariant under Galilean boosts and, for this reason, cannot be used as a metric. In order to make a boost-invariant combination one often considers the combination

$$
H_{\mu \nu}=E_{\mu}{ }^{a} E_{\nu}{ }^{b} \delta_{a b}+M_{\mu} \tau_{v}+M_{\nu} \tau_{\mu} .
$$

However, this combination is not invariant under central charge transformations. Nevertheless, it is used in the construction of a NR particle action coupled to NC gravity in such a way that the central charge gauge field $M_{\mu}$ couples to the particle via a Wess-Zumino (WZ) term of the form

$$
M_{\mu} \dot{x}^{\mu}
$$

where $x^{\mu}(\tau)$ is an embedding coordinate. This leads to a particle Lagrangian that is invariant under central charge transformations up to a total derivative. We will often call the symmetric tensor $H_{\mu \nu}$ the transverse metric and $\tau_{\mu \nu}$ the longitudinal metric. ${ }^{1}$

The central charge gauge field $M_{\mu}$ of NC gravity has a precursor in general relativity as an Abelian gauge field $\hat{M}_{\mu}$ to be added to general relativity. The only difference is that the Poincaré algebra does not get modified by the gauge field $\hat{M}_{\mu}$. This gauge field plays a crucial role in constructing NR limits without divergencies. For instance, starting from the Klein-Gordon Lagrangian coupled to general relativity one can only obtain the Schrödinger Lagrangian coupled to NC gravity as a NR limit provided one extends general relativity with a fluxless Abelian gauge field $\hat{M}_{\mu}$ that couples to a complex Klein-Gordon scalar. Similarly, one can only define a NR limit of a relativistic particle coupled to general relativity without divergencies provided the relativistic particle couples to $\hat{M}_{\mu}$ via a WZ term of the form

$$
\hat{M}_{\mu} \dot{x}^{\mu}
$$

It is instructive to give some details here. To define the NR limit we first express the Riemannian metric of general relativity and the gauge field $\hat{M}_{\mu}$ in terms of the NC fields (1) and a contraction parameter $\omega$. Next, after substituting these expressions into the action of the relativistic particle coupled to general relativity, we take the limit $\omega \rightarrow \infty$. This leads to a divergence linear in $\omega$ coming form the kinetic term that is cancelled by a similar divergent term coming from the WZ term by expressing $\hat{M}_{\mu}$ in terms of the NC fields as follows:

$$
\hat{M}_{\mu}=\omega \tau_{\mu}+\frac{1}{\omega} M_{\mu} .
$$

Given the fact that a vector field only couples via a WZ term to a particle, it is clear that one cannot apply the same procedure to define the NR limit of a string. In this case, it is the

\footnotetext{
${ }^{1}$ Strictly speaking, the metric $H_{\mu \nu}$ is only transverse in the absence of the terms containing the central charge gauge field $M_{\mu}$.
} 
Kalb-Ramond 2-form gauge field $\hat{B}_{\mu \nu}$ that couples to the relativistic string via a WZ term of the form

$$
\epsilon^{\alpha \beta} \partial_{\alpha} x^{\mu} \partial_{\beta} x^{\nu} \hat{B}_{\mu \nu}
$$

where $\partial_{\alpha}(\alpha=0,1)$ is the derivative with respect to the world-sheet coordinates $\sigma^{\alpha}$ and $x^{\mu}\left(\sigma^{\alpha}\right)$ are the string embedding coordinates. It turns out that taking the NR limit of a string leads to a divergence quadratic in $\omega$ coming from the kinetic term. To cancel this quadratic divergence we cannot work with a NC geometry since that contains only one clock function $\tau_{\mu}$ and there is no way to express the Kalb-Ramond field in terms of this single clock function. To cancel the quadratic divergence coming from the kinetic term we need two so-called longitudinal Vierbeine $\tau_{\mu}^{A}(A=0,1)$ and write

$$
\hat{B}_{\mu \nu}=\omega^{2} \epsilon_{A B} \tau_{\mu}{ }^{A} \tau_{\nu}{ }^{B}+B_{\mu \nu},
$$

where $B_{\mu \nu}$ is the NR Kalb-Ramond field. This leads to a new so-called String Newton-Cartan (SNC) geometry that is characterized by two special directions instead of the single Newtonian time direction in NC gravity. The difference between particles and strings is that a particle sweeps out a one-dimensional time direction whereas a sting sweeps out two directions longitudinal to the string: one time direction and one spatial direction, see Figure 2.

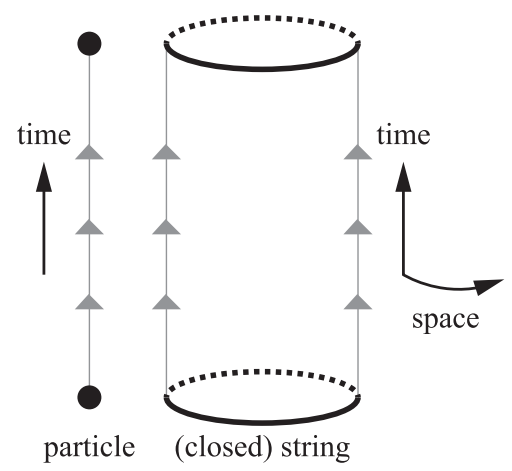

Figure 2: A particle (left) sweeps out a one-dimensional time direction whereas a string (right) sweeps out two directions: one time and one spatial direction.

Ignoring central extensions the algebra underlying the SNC geometry is the so-called string Galilei algebra where we distinguish between the two directions $A=0,1$ longitudinal to the string and the remaining directions $a=2, \cdots D-1$ transverse to the string. We thus have

$$
D \text { flat indices } \rightarrow\left\{\begin{array}{l}
2 \text { longitudinal indices A } \\
\text { D-2 transverse indices a }
\end{array}\right.
$$

with the following symmetries and generators:

$$
\begin{array}{rc}
\text { longitudinal translations } & H_{A} \\
\text { transverse translations } & P_{a} \\
\text { string Galilei boosts } & G_{A b} \\
\text { longitudinal Lorentz rotations } & J_{A B} \\
\text { transverse spatial rotations } & J_{a b}
\end{array}
$$


This string Galilei algebra is extended to a so-called enhanced string Galilei algebra with two types of non-central ${ }^{2}$ generators:

$$
Z_{A} \quad \text { and } \quad Z_{A B} \text { with } Z_{A}^{A}=0 .
$$

Ignoring matter fields, like the Kalb-Ramond 2-form field, the independent string NC fields are

$$
\left\{\tau_{\mu}{ }^{A}, E_{\mu}{ }^{a}, M_{\mu}{ }^{A}\right\} .
$$

For the construction of a NR string action we need both a longitudinal metric $\tau_{\mu \nu}$ and a transverse metric $H_{\mu \nu}$ which are the following generalizations of the particle case given in eqs. (3) and (5), respectively:

$$
\begin{aligned}
\text { longitudinal metric: } & \tau_{\mu \nu} \equiv \tau_{\mu}{ }^{A} \tau_{\nu}{ }^{B} \eta_{A B}, \\
\text { transverse metric: } & H_{\mu \nu} \equiv E_{\mu}{ }^{a} E_{\nu}{ }^{b} \delta_{a b}+\left(\tau_{\mu}{ }^{A} M_{\nu}{ }^{B}+\tau_{\nu}{ }^{A} M_{\mu}{ }^{B}\right) \eta_{A B} .
\end{aligned}
$$

\section{An Action for the NR Bosonic String}

We are now in a position to construct the action of NR string theory in a general SNC gravity background. For flat spacetime the action was already given a long time ago and reads $[2,3]$

$$
S_{\text {flat }}=-\frac{1}{4 \pi \alpha^{\prime}} \int d^{2} \sigma\left(\partial x^{a} \bar{\partial} x^{b} \delta_{a b}+\lambda \bar{\partial} X+\bar{\lambda} \partial \bar{X}\right),
$$

with

$$
X=x^{0}+x^{1}, \quad \bar{X}=x^{0}-x^{1}
$$

and similar for the Lagrange multipliers $\lambda, \bar{\lambda}$. A special feature of NR string theory is that the (perturbative) spectrum only contains winding strings along the compact $x^{1}$ direction [2].

The presence of the Lagrange multipliers can be understood as the result of taking the NR limit of the relativistic string action in Polyakov form. ${ }^{3}$ This is best understood by comparing to the particle and considering the following relativistic particle action coupled to general relativity in Polyakov form:

$$
S_{\text {Pol. }}=-\frac{1}{2} \int d \tau\left\{-\frac{1}{e} \hat{E}_{\mu} \hat{A}^{\mu} \hat{x}^{\mu} \hat{E}_{\nu} \dot{x}^{\nu} \eta_{\hat{A} \hat{B}}+M^{2} e-2 M \hat{M}_{\mu} \dot{x}^{\mu}\right\} .
$$

Here $e$ is the worldline Einbein and $M$ is a mass parameter. Expanding the general relativity fields in terms of the Newton-Cartan background fields one encounters the following quadratic divergence that is not cancelled by the vector field in the Wess-Zumino term:

$$
S_{\text {Pol. }}\left(\omega^{2}\right)=-\frac{1}{2} \int d \tau \frac{1}{e} \omega^{2}\left[\tau_{\mu} \dot{x}^{\mu}-m e\right]^{2} .
$$

It should be noted that this is an artefact of the Polyakov formulation. In the Nambu-Goto formulation there is no quadratic divergence left. The quadratic divergence given in (16) is not fatal. The reason for this is that it is the square of something and therefore can be rewritten, using a Lagrange multiplier $\lambda$ as follows:

$$
S_{\text {Pol. }}\left(\omega^{2}\right)=-\frac{1}{2} \int d \tau \frac{1}{e}\left\{\lambda\left(\tau_{\mu} \dot{x}^{\mu}-m e\right)-\frac{1}{4 \omega^{2}} \lambda^{2}\right\} .
$$

\footnotetext{
${ }^{2}$ We call a generator non-central if it only has non-zero commutators due to its index structure.

${ }^{3}$ The presence of the Lagrange multipliers can alternatively be understood by taking the NR limit in an Hamiltonian formulation.
} 
Written in this form, the limit that $\omega \rightarrow \infty$ can be taken and one ends up with the following NR Polyakov action:

$$
S_{\text {Pol. }}(\text { N.R. })=-\frac{1}{2} \int d \tau \frac{1}{e}\left\{\dot{x}^{\mu} \dot{x}^{\nu} H_{\mu \nu}+\lambda\left(\tau_{\mu} \dot{x}^{\mu}-m e\right)\right\} .
$$

Integrating out the Lagrange multiplier $\lambda$ one finds that

$$
e=\frac{\tau_{\mu} \dot{x}^{\mu}}{m} \text {. }
$$

Substituting this back into the Polyakov action (18) one obtains the following NR particle action in Nambu-Goto form:

$$
S_{\text {N.G. }}(\text { N.R. })=-\frac{m}{2} \int d \tau \frac{\dot{x}^{\mu} \dot{x}^{v}}{\tau_{\rho} \dot{x}^{\rho}} H_{\mu \nu} .
$$

One can now take a similar limit of the relativistic Polyakov string. We thus find the following expression for a NR string in a (matter-coupled) SNC background $[4,5]:{ }^{4}$

$$
\begin{aligned}
S_{\mathrm{SNC}}= & -\frac{T}{2} \int d^{2} \sigma\left[\sqrt{-h} h^{\alpha \beta} \partial_{\alpha} x^{\mu} \partial_{\beta} x^{\nu} H_{\mu \nu}+\epsilon^{\alpha \beta}\left(\lambda e_{\alpha} \tau_{\mu}+\bar{\lambda}_{\bar{e}_{\alpha}} \bar{\tau}_{\mu}\right) \partial_{\beta} x^{\mu}\right] \\
& -\frac{T}{2} \int d^{2} \sigma \epsilon^{\alpha \beta} \partial_{\alpha} x^{\mu} \partial_{\beta} x^{\nu} B_{\mu \nu}+\frac{1}{4 \pi} \int d^{2} \sigma \sqrt{-h} R\left(\Phi-\frac{1}{4} \ln G\right),
\end{aligned}
$$

where $T$ is the string tension, $\sigma^{\alpha}$ are the world-sheet coordinates, $h_{\alpha \beta}=e_{\alpha}{ }^{a} e_{\beta}{ }^{b} \eta_{a b}$ is the worldsheet metric with Zweibeine $e_{\alpha}{ }^{a}, R^{(2)}$ is the Ricci scalar defined with respect to $h_{\alpha \beta}$ and $x^{\mu}(\sigma), \mu=0,1, \cdots, D-1$ are the string embedding coordinates. The action (21) also describes the coupling to the background Kalb-Ramond field $B_{\mu \nu}$ and the dilaton $\Phi$. Furthermore, $\lambda$ and $\bar{\lambda}$ are two world-sheet Lagrange multiplier fields whose equations of motion allow us to solve for the world-sheet metric $h_{\alpha \beta}$ up tp a scale factor $\alpha(x)$ in terms of the pullback of the longitudinal metric $\tau_{\mu \nu}$ as follows:

$$
h_{\alpha \beta}=\alpha(x) \partial_{\alpha} x^{\mu} \partial_{\beta} x^{v} \tau_{\mu \nu} .
$$

As mentioned in the previous section, the so-called transverse metric $H_{\mu \nu}$ is given in terms of the SNC background fields by ${ }^{5}$

$$
H_{\mu \nu}=E_{\mu}{ }^{a} E_{\nu}{ }^{b} \delta_{a b}+\left(\tau_{\mu}{ }^{A} M_{\nu}{ }^{B}+\tau_{\nu}{ }^{A} M_{\mu}{ }^{B}\right) \eta_{A B} .
$$

The definition of $G$ occurring in the string sigma model action (21) in terms of $H_{\mu \nu}$ and $\tau_{\mu}{ }^{A}$ is given by

$$
G=\operatorname{det} H_{\mu \nu} \operatorname{det}\left(\tau_{\rho}^{A} H^{\rho \sigma} \tau_{\sigma}^{B}\right) .
$$

Finally, the lightcone components $\tau_{\mu}, \bar{\tau}_{\mu}$ of $\tau_{\mu}{ }^{A}$ and $e_{\alpha}, \bar{e}_{\alpha}$ of $e_{\alpha}{ }^{a}$ are defined in [4,5].

Upon integrating out the Lagrange multipliers, one can show that the string action is invariant under Galilean boosts with parameters $\lambda^{A a}$, non-central charge transformations with parameters $\lambda^{A}$ and second non-central charge transformations with parameters $\sigma_{B}^{A}$ (with $\left.\sigma_{A}^{A}=0\right)$ :

$$
\begin{aligned}
\delta \tau_{\mu}{ }^{A} & =0, \\
\delta E_{\mu}{ }^{a} & =-\lambda_{A}{ }^{a} \tau_{\mu}{ }^{A}, \\
\delta M_{\mu}{ }^{A} & =D_{\mu}(\omega) \lambda^{A}+\lambda^{A}{ }_{a} E_{\mu}{ }^{a}+\sigma^{A}{ }_{B} \tau_{\mu}{ }^{B} .
\end{aligned}
$$

\footnotetext{
${ }^{4}$ For other recent work on non-relativistic strings in a curved background, see [6-12].

${ }^{5}$ Note that this metric is strictly speaking transverse only in the absence of the second term.
} 
Here $D_{\mu}(\omega)$ is the Lorentz-covariant derivative with respect to the longitudinal Lorentz rotations. Note that the gauge field corresponding to the second non-central charge transformation does not occur in the string action. The invariance under the first non-central charge transformations is valid provided that the following zero torsion constraint holds: ${ }^{6}$

$$
D_{[\mu}(\omega) \tau_{\nu]}^{A}=0 .
$$

Part of this constraint contains the spin-connection field $\omega_{\mu}{ }^{A B}$, enabling one to solve this connection field in terms of $\tau_{\mu}{ }^{A}$ and its derivative. The remaining part is a geometric constraint given by the projection of (26) that does not contain the spin-connection:

$$
\epsilon_{C}{ }^{\left(A^{A}\right.} \tau_{[\mu}^{B)} \partial_{\nu} \tau_{\rho]}^{C}=0 .
$$

An important feature of the NR action (21), which is absent in the relativistic case, is that the action is invariant under certain Stückelberg symmetries of the background fields implying that some of the components only occur in special combinations. A similar thing happens for the NR Nambu-Goto particle coupled to a vector gauge field $B_{\mu}$ :

$$
S_{\mathrm{NG}} \text { (N.R.) }=-\frac{m}{2} \int d \tau\left\{\frac{\dot{x}^{\mu} \dot{x}^{\nu}}{\tau_{\rho} \dot{x}^{\rho}} H_{\mu \nu}-B_{\mu} \dot{x}^{\mu}\right\},
$$

in which case the Stückelberg symmetries are given by

$$
H_{\mu \nu} \rightarrow H_{\mu \nu}+\frac{1}{2}\left(\tau_{\mu} C_{v}+\tau_{\nu} C_{\mu}\right), \quad B_{\mu} \rightarrow C_{\mu} .
$$

In terms of the Stückelberg-invariant combinations the NR particle action (28) reads

$$
S_{\mathrm{NG}}(\mathrm{N} . \mathrm{R} .)=-\frac{m}{2} \int d \tau\left\{\frac{E^{a} E^{b} \delta_{a b}}{\tau}+\tau\left(H_{00}-B_{0}\right)+E^{a}\left(H_{0 a}-B_{a}\right)\right\},
$$

where we have used flat indices and where we have defined

$$
\tau \equiv \dot{x}^{\mu} \tau_{\mu}, \quad E^{a} \equiv \dot{x}^{\mu} E_{\mu}{ }^{a} .
$$

Similarly, one finds that, after integrating out the Lagrange multipliers, the NR string action (21) is invariant under the following (infinitesimal) Stueckelberg symmetries, with parameters $C_{\mu}{ }^{A}$, given by

$$
\delta B_{\mu \nu}=\left(C_{\mu}{ }^{A} \tau_{\nu}^{B}-C_{\nu}{ }^{A} \tau_{\mu}{ }^{B}\right) \epsilon_{A B}, \quad \delta m_{\mu}{ }^{A}=-C_{\mu}{ }^{A} .
$$

This Stueckelberg symmetry is a reducible symmetry in the sense that the transformation rule (32) of $B_{\mu \nu}$ is formally invariant under a gauge symmetry, with singlet parameter $C$, given by

$$
\delta C_{\mu}{ }^{A}=\epsilon^{A B} \tau_{\mu B} C .
$$

\section{Discussion}

Once the action for the NR string in a curved background has been constructed several research directions become possible. Following the techniques of $[13,14]$ we have constructed a NR version of the T-duality rules $[4,5]$. A remarkable consequence of this T-duality is that taking

\footnotetext{
${ }^{6}$ At the classical level there is another way to achieve invariance of the action under the first non-central charge transformations by assigning to the Kalb-Ramond field an extra central charge transformation that is proportional to the torsion [12].
} 
the T-dual along the spatial direction of the string leads to a string theory that looks relativistic but in fact, due to the presence of a null-isometry, is non-relativistic. The (one-loop) beta functions of the string sigma model, leading to field equations of the background fields, have been calculated both for the closed string $[15,16]$ as well as for the open string [17]. An intriguing consequence of the Stueckelberg symmetries mentioned in section 3 is that there are less equations of motion than in the relativistic case. The missing equations of motion are precisely in the same representation as the Stueckelberg parameters. ${ }^{7}$

An interesting future research direction is to generalize the results of [19] on superstrings in a flat background and of [18] on superstrings in a special curved background to superstrings in a general curved background and to see what the geometry is that one is ending up with. This would open the way to start discussing NR D-branes and NR holography from the perspective of a NR gravity theory in the bulk. We hope to come back to these interesting research equations in the nearby future.

\section{Acknowledgements}

This talk was based upon the papers [4,5]. We thank our collaborators for the many stimulating discussions we had with them. We also thank the organizers of this on-line conference for creating this special opportunity.

\section{References}

[1] I. Ciufolini, D. Dominici and L. Lusanna, 2001: A relativistic spacetime odyssey, Bronstein M 1933 Uspekhi Astronomicheskikh Nauk. Sbornik 3, World Scientific (2003), doi:10.1142/5040 .

[2] J. Gomis and H. Ooguri, Nonrelativistic closed string theory, J. Math. Phys. 42, 3127 (2001), doi:10.1063/1.1372697.

[3] U. H. Danielsson, M. Kruczenski and A. Güijosa, IIA/B, wound and wrapped, J. High Energ. Phys. 10, 020 (2000), doi:10.1088/1126-6708/2000/10/020.

[4] E. Bergshoeff, J. Gomis and Z. Yan, Nonrelativistic string theory and T-duality, J. High Energ. Phys. 11, 133 (2018), doi:10.1007/JHEP11(2018)133.

[5] E. A. Bergshoeff, J. Gomis, J. Rosseel, C. Şimşek and Z. Yan, String theory and string Newton-Cartan geometry, J. Phys. A: Math. Theor. 53, 014001 (2020), doi:10.1088/17518121/ab56e9.

[6] T. Harmark, J. Hartong and N. A. Obers, Nonrelativistic strings and limits of the AdS/CFT correspondence, Phys. Rev. D 96, 086019 (2017), doi:10.1103/PhysRevD.96.086019.

[7] J. Klusoň, Remark about non-relativistic string in Newton-Cartan background and null reduction, J. High Energ. Phys. 05, 041 (2018), doi:10.1007/JHEP05(2018)041.

[8] T. Harmark, J. Hartong, L. Menculini, N. A. Obers and Z. Yan, Strings with non-relativistic conformal symmetry and limits of the AdS/CFT correspondence, J. High Energ. Phys. 11, 190 (2018), doi:10.1007/JHEP11(2018)190.

\footnotetext{
${ }^{7}$ This counting only works if we use the fact that the Stueckelberg symmetries (32) are reducible, see eq. (33), and therefore effectively have one singlet parameter less.
} 
[9] J. Klusoň, Note about T-duality of non-relativistic string, J. High Energ. Phys. 08, 074 (2019), doi:10.1007/JHEP08(2019)074.

[10] J. Klusoň, ( $m, n$ )-string and D1-brane in stringy Newton-Cartan background, J. High Energ. Phys. 04, 163 (2019), doi:10.1007/JHEP04(2019)163.

[11] D. Roychowdhury, Probing tachyon kinks in Newton-Cartan background, Phys. Lett. B 795, 225 (2019), doi:10.1016/j.physletb.2019.06.031.

[12] T. Harmark, J. Hartong, L. Menculini, N. A. Obers and G. Oling, Relating non-relativistic string theories, J. High Energ. Phys. 11, 071 (2019), doi:10.1007/JHEP11(2019)071.

[13] T. H. Buscher, Path-integral derivation of quantum duality in nonlinear sigma-models, Phys. Lett. B 201, 466 (1988), doi:10.1016/0370-2693(88)90602-8.

[14] T. H. Buscher, A symmetry of the string background field equations, Phys. Lett. B 194, 59 (1987), doi:10.1016/0370-2693(87)90769-6.

[15] J. Gomis, J. Oh and Z. Yan, Nonrelativistic string theory in background fields, J. High Energ. Phys. 10, 101 (2019), doi:10.1007/JHEP10(2019)101.

[16] Z. Yan and M. Yu, Background field method for nonlinear sigma models in nonrelativistic string theory, J. High Energ. Phys. 03, 181 (2020), doi:10.1007/JHEP03(2020)181.

[17] J. Gomis, Z. Yan and M. Yu, T-duality in nonrelativistic open string theory (2020), arXiv:2008.05493.

[18] J. Gomis, J. Gomis and K. Kamimura, Non-relativistic superstrings: a new soluble sector of $A d S_{5} \times S^{5}$, J. High Energ. Phys. 12, 024 (2005), doi:10.1088/1126-6708/2005/12/024.

[19] J. Gomis, K. Kamimura and P. K. Townsend, Non-relativistic superbranes, J. High Energ. Phys. 11, 051 (2004), doi:10.1088/1126-6708/2004/11/051. 